\title{
Avalanche in the Valley \\ (Fermions, Anomaly and Unitarity in High-Energy Electroweak Interactions)
}

\author{
Riccardo Guida and Kenichi Konishi \\ Dipartimento di Fisica - Università di Genova \\ Istituto Nazionale di Fisica Nucleare - sez. di Genova \\ Via Dodecaneso, 33 - 16146 Genova (Italy) \\ E-mail: Decnet 39166; Bitnet@GENOVA.INFN.IT
}

\begin{abstract}
Problems related to fermions, unitarity and chiral anomaly in high energy electroweak interactions, are investigated. Particular attention is paid to the correct functional integration over fermion fields in the background of instanton-antiinstanton type configurations. This leads to an expansion of correlation functions in terms of a small parameter, $\rho / R$, when the instanton-antiinstanton separation $(R)$ is large compared to their sizes $(\rho)$. Applying such a method to widely discussed cases of fermion-number violation in the electroweak theory, we conclude that there are no theoretical basis for expecting anomalous cross sections to become observable at energies in the $10 \mathrm{TeV}$ region.
\end{abstract}

GEF-Th-16/1992

October 1992 
In spite of much effort, the question whether instanton- (or sphaleron-) induced electroweak interactions become strong in high energy scattering processes, remains unsettled[1-20]. There are arguments, which use unitarity constraints on multiple gauge-boson production processes $[8,9,11,13,18]$, that such processes are always exponentially suppressed by a finite fraction of the 't Hooft's factor. These arguments are quite convincing in our opinion; however it appears difficult to make them more quantitative.

Both direct calculation of the cross section by instanton method, as in the original calculation of Refs.[3,5,7], and another approach $[6,8,12,15]$ which makes use of the optical theorem and the so-called valley method, encounter various technical difficulties. In some model calculations within the second approach (see the second of Ref.[12]), it was claimed that the fermion number violating cross section approaches the geometrical one at energies of order of the sphaleron mass.

One point in the second approach ( based on the optical theorem), however, has always been a little obscure to us. It seems that the functional integration over fermions in the instanton-antiinstanton background, has never been done properly. The "fermion part of the valley trajectory" mentioned in Ref.[12] can easily be shown to be inconsistent [19]; the projection operator introduced in Ref.[6] was just put in by hand and no calculation was really done with it. We wish to clarify some of these and related issues in this paper.

The problem to be resolved turns out to be one of quite general nature. Namely: how is unitarity obeyed in the presence of topologically nontrivial effects such as instantons, treated within the semiclassical approximation?*

The optical theorem states that the cross section,

$$
1+2 \longrightarrow X
$$

summed over $X$, is equal, apart from a kinematical factor, to the imaginary part of the forward elastic amplitude,

$$
1+2 \longrightarrow 1+2
$$

Now consider a particular class of processes (1) induced by an instanton, with the change of the fermion number,

$$
\Delta f=f_{1}+f_{2}-f_{X}=N_{F},
$$

* The work of Ref.[10] (see also Ref.[16]) goes quite some way in proving unitarity in such a situation, especially as regards the final states (i.e., states summed over in the unitarity relations). However, fermions are not considered there: as a result no discussion on subtleties related to chiral anomaly is found in [10]. Note that even disregarding fermions, a problem arises with the external (or "initial") particles anyway. See the last paragraph of this paper. 
where $N_{F}$ is the number of the lefthanded $S U(2)$ doublets in the theory. The sum over the final states satisfying (3) should be a part of the imaginary part of the elastic amplitude.

For an (anti)instanton background, which is relevant for the calculation of the production process (1), each right (left) handed fermion field has a zero mode. The standard functional integration over fermions yields a product of these zero modes; by going to momentum space and by applying the LSZ amputation one finds the S-matrix elements consistent with (3).

The corresponding contribution in the elastic amplitude (2) must arise from a sort of instanton-antiinstanton $(i-a)$ background, topologically (globally) equivalent to the trivial, perturbative vacuum. One would expect however that no fermion zero modes exist in such a background (see below for more detail). How then can one extract the "anomalous" part of the elastic amplitude?

To make the problem well-defined, we work with a particular class of $i-a$ type configurations - the so called valley, or streamline, trajectory $[22,12] *$

$$
\begin{aligned}
& A_{\mu}^{(\text {valley })}=-\frac{i}{g}\left(\sigma_{\mu} \bar{\sigma}_{\nu}-\delta_{\mu \nu}\right)\left[\frac{\left(x-x_{a}\right)_{\nu}}{\left(x-x_{a}\right)^{2}+\rho^{2}}+\frac{\left(x-x_{i}\right)_{\nu} \rho^{2}}{\left(x-x_{i}\right)^{2}\left(\left(x-x_{i}\right)^{2}+\rho^{2}\right)}\right. \\
& \left.+\frac{\left(x-x_{i}+y\right)_{\nu}}{\left(x-x_{i}+y\right)^{2}}-\frac{\left(x-x_{i}\right)_{\nu}}{\left(x-x_{i}\right)^{2}}\right] ; \\
& y=-R /(z-1) ; z=\left(R^{2}+2 \rho^{2}+\sqrt{R^{4}+4 \rho^{2} R^{2}}\right) / 2 \rho^{2} ; R^{\mu}=\left(x_{i}-x_{a}\right)^{\mu}
\end{aligned}
$$

which is known to have the correct properties at large $R$ at least $[7,10,12]$ to reproduce the square of the amplitude (1). The classical field Eq.(4) interpolates two solutions of the Euclidean field equations, $A_{\mu}=A_{\mu}^{(i)}+A_{\mu}^{(a)}\left(\right.$ at $\left.R=\left|x_{a}-x_{i}\right|=\infty\right)$ and a gauge-equivalent of $A_{\mu}=0$ (at $R=0$ ). The instanton appears in the singular gauge while the antiinstanton is in the regular one. Moreover for simplicity we have set $\rho_{a}=\rho_{i}=\rho$, in Eq.(4). Importance of the non-Gaussian integrations along such an almost flat valley, was emphasised first in Ref.[21,22] in a general context of quantum mechanics and QCD.

We wish to compute the four point function,

$$
<T \psi_{1}(x) \psi_{2}(u) \bar{\psi}_{1}(y) \bar{\psi}_{2}(v)>^{\left(A_{\text {valley }}\right)}
$$

* Here are some symbols and conventions used below: $\bar{D} \equiv D_{\mu} \bar{\sigma}_{\mu} ; \bar{\sigma}_{\mu} \equiv\left(-i, \sigma^{a}\right)$; $a=1,2,3 . D \equiv D_{\mu} \sigma_{\mu}$, where $\sigma_{\mu} \equiv\left(i, \sigma^{a}\right)$. Unless indicated by a superscrpt $a$ (antiinstanton) or $i$ (instanton), the covariant derivative will refer to the valley background of Eq. (4). The spinor indices as in $\left(\sigma_{\mu}\right)_{\alpha \dot{\alpha}}$ and $\left(\bar{\sigma}_{\mu}\right)^{\dot{\alpha} \alpha}$ are suppressed. The fermion fields $\psi_{j}$ are left-handed ones. Throughout the paper, we work in the Euclidean spacetime: the continuation to the Minkowski spacetime of the final formulae is understood. 


$$
\begin{gathered}
=\int \mathcal{D} \psi \mathcal{D} \bar{\psi} \psi_{1}(x) \psi_{2}(u) \bar{\psi}_{1}(y) \bar{\psi}_{2}(v) \mathrm{e}^{-S} / Z^{(A=0)} \\
S=\sum_{j=1}^{N_{F}} \int d^{4} x i \bar{\psi}_{j} \bar{D} \psi_{j}
\end{gathered}
$$

in the fixed background of Eq.(4). Integrations over the collective coordinates such as $R$ and $\rho$ are to be performed afterwards.

As the functional integral factorises in flavour we must study (suppressing the flavour index),

$$
I(x, y)=\int \mathcal{D} \psi \mathcal{D} \bar{\psi} \psi(x) \bar{\psi}(y) \exp -\int d^{4} x i \bar{\psi} \bar{D} \psi
$$

and

$$
\mathcal{Z}=\int \mathcal{D} \psi \mathcal{D} \bar{\psi} \exp -\int d^{4} x i \bar{\psi} \bar{D} \psi=\operatorname{det} \bar{D}
$$

Large $R / \rho$.

At large $R$ one expects the generating functional to reduce to the product $\mathcal{Z}^{(a)} \cdot \mathcal{Z}^{(i)}$, where $\mathcal{Z}^{(a)}\left(\mathcal{Z}^{(i)}\right)$ is the generating functional in the pure antiinstanton (instanton) background. To see this, let us introduce complete sets of orthonormal modes $\left\{\eta_{n}^{(a)}\right\}$ and $\left\{\bar{\zeta}_{n}^{(i)}\right\}, n=0,1,2, \ldots$, for the left-handed and right-handed fermions, respectively. They are eigenstates of $D^{(a)} \bar{D}^{(a)}$ and $\bar{D}^{(i)} D^{(i)}$ :

$$
\begin{array}{cl}
\bar{D}^{(a)} \eta_{m}^{(a)}=\bar{k}_{m} \bar{\zeta}_{m}^{(a)}(m=0,1, \ldots), & D^{(a)} \bar{\zeta}_{m}^{(a)}=k_{m} \eta_{m}^{(a)}(m=1,2, \ldots), \\
\bar{D}^{(i)} \eta_{m}^{(i)}=l_{m} \bar{\zeta}_{m}^{(i)}(m=1,2, \ldots), & D^{(i)} \bar{\zeta}_{m}^{(i)}=\bar{l}_{m} \eta_{m}^{(i)}(m=0,1, \ldots),
\end{array}
$$

where $\bar{k}_{0}=\bar{l}_{0}=0$. The functional integral is then defined as:

$$
\begin{gathered}
\int \mathcal{D} \psi \mathcal{D} \bar{\psi} \equiv \prod_{m, n=0} d a_{m} d \bar{b}_{n} \\
\psi=\sum_{m=0}^{\infty} a_{m} \eta_{m}^{(a)}, \quad \bar{\psi}=\sum_{n=0}^{\infty} \bar{b}_{n} \bar{\zeta}_{n}^{(i) *}
\end{gathered}
$$

Now the two point function $I(x, y)$ can be written as:

$$
\begin{aligned}
I(x, y) & =\operatorname{det} \bar{D}\left\langle x\left|\bar{D}^{-1}\right| y\right\rangle \\
& =\operatorname{det} \bar{D}\left\{\langle x \mid a, 0\rangle\left\langle a, 0\left|\bar{D}^{-1}\right| i, 0\right\rangle\langle i, 0 \mid y\rangle+\sum_{m \neq 0}\langle x \mid a, m\rangle\left\langle a, m\left|\bar{D}^{-1}\right| i, 0\right\rangle\langle i, 0 \mid y\rangle\right. \\
& \left.+\sum_{n \neq 0}\langle x \mid a, 0\rangle\left\langle a, 0\left|\bar{D}^{-1}\right| i, n\right\rangle\langle i, n \mid y\rangle+\sum_{m, n \neq 0}\langle x \mid a, m\rangle\left\langle a, m\left|\bar{D}^{-1}\right| i, n\right\rangle\langle i, n \mid y\rangle\right\}:
\end{aligned}
$$


the term proportional to the product of the zero modes has been singled out. We wish to compute Eq.(8) as an expansion in the small ratio $\rho / R$. To do this, we first note that the matrix $\bar{D}$ has the following characteristic structure. We write

$$
\bar{D}=\left(\begin{array}{ccccc}
d & v_{1} & \ldots & v_{n} & \ldots \\
w_{1} & X_{11} & \ldots & X_{1 n} & \ldots \\
\vdots & \vdots & \ddots & \vdots & \ddots \\
w_{m} & X_{m 1} & \ldots & X_{m n} & \ldots \\
\vdots & \vdots & \ddots & \vdots & \ddots
\end{array}\right)
$$

where $d \equiv(\bar{D})_{00}=\langle i, 0|\bar{D}| a, 0\rangle=\langle i, 0|\bar{C}| a, 0\rangle, v_{n} \equiv(\bar{D})_{0 n}=\langle i, 0|\bar{D}| a, n\rangle=$ $\langle i, 0|\bar{B}| a, n\rangle$ and $w_{m}=\langle i, m|\bar{D}| a, 0\rangle=\langle i, m|\bar{C}| a, 0\rangle .{ }^{*}$

The inverse matrix $\bar{D}^{-1}$ is given by:

$$
\begin{aligned}
\left(\bar{D}^{-1}\right)_{00} & =1 /\left(d-v X^{-1} w\right) \\
& =d^{-1}+d^{-2} v_{m}\left(X^{-1}\right)_{m n} w_{n}+\cdots ; \\
\left(\bar{D}^{-1}\right)_{m n} & =\left(X-d^{-1} w \otimes v\right)^{-1}=X^{-1}\left(\mathbf{1}-d^{-1} w \otimes v X^{-1}\right)^{-1} \\
& =\left(X^{-1}\right)_{m n}+d^{-1}\left(X^{-1}\right)_{m l} w_{l} v_{k}\left(X^{-1}\right)_{k n}+\cdots, \\
\left(\bar{D}^{-1}\right)_{0 n} & =-d^{-1} v_{l}\left(\bar{D}^{-1}\right)_{l n}, \\
\left(\bar{D}^{-1}\right)_{m 0} & =-\left(\bar{D}^{-1}\right)_{00} X_{m k}^{-1} w_{k},
\end{aligned}
$$

where $X^{-1}$ is the inverse of the submatrix $X$.

Inserting Eq.(10) in Eq.(8) and after some algebra we find a simple expression for $I(x, y)[19]$ :

$$
\begin{aligned}
I(x, y) & =\operatorname{det} X\left\{\langle x \mid a, 0\rangle-\left\langle x\left|X^{-1} \bar{C}\right| a, 0\right\rangle\right\}\left\{\langle i, 0 \mid y\rangle-\left\langle i, 0\left|\bar{B} X^{-1}\right| y\right\rangle\right\} \\
& +\operatorname{det} \bar{D}\left\langle x\left|X^{-1}\right| y\right\rangle .
\end{aligned}
$$

Furthermore $X^{-1}$ can itself be expressed as [19],

$$
X^{-1}=\left(\mathbf{1}+\bar{S}\left(\bar{C}-\mathcal{Q}_{i} \bar{B}\right)\right)^{-1} \bar{S} \mathcal{P}_{i}
$$

where $\bar{S}$ is the "propagator" in the anti-instanton background [23]:

$$
\langle x|\bar{S}| y\rangle=\sum_{m \neq 0} \eta_{m}^{(a)}(x) \bar{\zeta}_{m}^{(a)}(y)^{*} / \bar{k}_{m} ; \quad \bar{D}^{(a)} \bar{S}=\mathbf{1} ; \quad \bar{S} \bar{D}^{(a)}=\mathcal{P}_{a}
$$

\footnotetext{
* $\bar{C}$ and $\bar{B}$ are defined as $\bar{D}=\bar{D}^{(a)}+\bar{C}=\bar{D}^{(i)}+\bar{B}$, where $\bar{D}^{(a)}$ and $\bar{D}^{(i)}$ are the covariant derivatives in the pure antiinstanton (or instanton) background, and $\bar{D}^{(a)}|a, 0\rangle=0 ;\langle i, 0| \bar{D}^{(i)}=0$. The asymptotic behaviour, $\eta_{0}^{(a)} \sim \frac{\rho}{\left(x-x_{a}\right)^{3}} ; \bar{\zeta}_{0}^{(i)} \sim$ $\frac{\rho}{\left(x-x_{i}\right)^{3}} ; \bar{C}(x) \sim \frac{\rho^{2}}{\left(x-x_{i}\right)^{3}} ; \bar{B}(x) \sim \frac{1}{x-x_{a}}$, can be used to estimate various matrix elements below.
} 
and

$$
\mathcal{P}_{i} \equiv \mathbf{1}-\mathcal{Q}_{i} ; \quad \mathcal{Q}_{i} \equiv|i, 0\rangle\left\langle i, 0\left|; \quad \mathcal{P}_{a} \equiv \mathbf{1}-\mathcal{Q}_{a} ; \quad \mathcal{Q}_{a} \equiv\right| a, 0\right\rangle\langle a, 0|,
$$

are projection operators.

Using the above expression of $X^{-1}$ in Eq.(11) we arrive, after some reshuffling, at our final (and still exact) result for the two point function:

$$
\begin{aligned}
& I(x, y)=\{\operatorname{det} X /(1-f)\}\langle x|(\mathbf{1}-\mathcal{G} \bar{C})| a, 0\rangle\langle i, 0|(\mathbf{1}-\bar{B} \mathcal{G})| y\rangle \\
&+\operatorname{det} \bar{D}\langle x|\mathcal{G}| y\rangle ; \\
& \mathcal{G} \equiv \bar{S}(\mathbf{1}+\bar{C} \bar{S})^{-1} ; f \equiv\langle i, 0|\bar{B} \mathcal{G}| i, 0\rangle .
\end{aligned}
$$

By expanding $\mathcal{G}$ as $\mathcal{G}=\bar{S}-\bar{S} \bar{C} \bar{S}+\cdots$ we obtain the "overlap expansion" of $I(x, y)$ in the small parameter $\rho / R$. The reason for that is that products such as $\bar{C}(z) \eta_{0}^{(a)}(z)$ and $\bar{B}(z) \bar{\zeta}_{0}^{(i)}(z)$ with small overlapping support appear in the integrals. It is also easy to see by using the explicit expression of the propagator $\bar{S}$ [23] that an extra power of $\bar{S} \bar{C}$ gives rise to a further suppression. Some useful results are:

$$
\begin{aligned}
d & =\bar{D}_{00}=\int_{z} \bar{\zeta}_{0}^{(i)}(z)^{*} \bar{C}(z) \eta_{0}^{(a)}(z) \sim \rho^{2} / R^{3} ; \\
\left(\bar{D}^{-1}\right)_{00} & =(1-f)(d-\langle i, 0|\bar{B} \mathcal{G} \bar{C}| a, 0\rangle)^{-1} \sim d^{-1} ; \\
\operatorname{det} \bar{D} & =\left(\left(\bar{D}^{-1}\right)_{00}\right)^{-1} \operatorname{det} X \simeq \text { const. } \rho^{2} / R^{3} .
\end{aligned}
$$

Now, the "anomalous" part of the elastic amplitude must arise from those terms in Eq.(12) which have the factorised dependence on $x$ and $y$, i.e., terms of the form, $\sim F(x) G(y)$. The reason is that the initial fermion at $y$ must not be connected to the final fermion at $x$ by a chain of propagators: it must be absorbed by the instanton. In the semi- classical approximation this means factorisation.

All terms arising from the expansion of the first term of $I(x, y)$ in Eq.(12) have such a factorised structure. The non-factorised, "non-anomalous" contribution comes from the second term.

A detailed analysis of Eq.(12) allows us to show [19] then that the anomalous part of $I(x, y)$ is essentially dominated at large $R / \rho$ by

$$
I^{(\text {anom })}(x, y) \simeq \eta_{0}^{(a)}(x) \bar{\zeta}_{0}^{(i)}(y)^{*}+\cdots,
$$

which originates in the first term of Eq.(8). Inserted in Eq.(5), it leads upon LSZ reduction * to the anomalous part of the elastic amplitude, required by unitarity.

\footnotetext{
* Some care in the choice of gauge must be taken in the LSZ procedure; see [19].
} 


\section{Fermions in the intermediate state}

To reach the above conclusion truely, however, we must make one further check. For the leading term of Eq.(14) to represent the anomalous process, the intermediate state must contain fermions satisfying the selection rule, Eq.(3).

That this is indeed so can be seen from Eq.(13). The functional integration yields, for each flavour $\left(i=3,4, \cdots N_{F}\right)$ a factor

$$
\operatorname{det} \bar{D} / \operatorname{det} \bar{\partial} \sim \rho^{2} / R^{3}
$$

but this is precisely the factor expected for a left-handed fermion, produced at the antiinstanton center (with amplitude $\rho$ ), propagating freely backward to the instanton (with amplitude, $\sim 1 /\left(x_{a}-x_{i}\right)^{3}=1 / R^{3}$ ) and absorbed by the latter (with amplitude, $\rho$ ).

To be even more explicit, suppose that we are going to observe the intermediate state by setting up an appropriate detector. This would correspond to introducing a source (or sink) term for each flavour, $\int d x J_{\mu}^{i}(x) \psi_{i}(x)+h . c$. , and taking the first derivative with respect to the sources. This would produce pairs of zero modes (as in Eq.(14)): the fermions required by the topological selection rule are indeed there, as long as $R / \rho$ is large.

\section{Small $R / \rho$.}

The overlap expansion of Eq.(12) fails at small $R / \rho \leq 1$ for obvious reasons. In particular, in the limit $R / \rho \rightarrow 0$, the classical field $A_{\mu}^{(\text {valley })}$ reduces to the trivial, perturbative vacuum. In the free theory, the procedure adopted above is still formally valid, but the appearance of the product of the zero modes (as in Eq.(14)) is of course a fake, the total two-point function being simply $\sigma_{\mu}(x-y)_{\mu} /(x-y)^{4}$. This means that at certain $R / \rho \sim O(1)$ the first term of Eq.(14), corresponding to the anomalous process, must be effectively cancelled by contributions from other terms (left implicit in Eq.(14)). It probably does not make sense to ask exactly at which value of $R / \rho$ this occurs, but there is a fairly good evidence that such a transition takes place rather abruptly.

We have in fact computed numerically the integral of the topological density,

$$
C\left(x_{4}\right)=-\int_{-\infty}^{x_{4}} \int d^{3} x \frac{g^{2}}{16 \pi^{2}} \operatorname{Tr}\left(F_{\mu \nu} \tilde{F}_{\mu \nu}\right)=\mathcal{N}_{C S}\left(x_{4}\right)-\mathcal{N}_{C S}(-\infty)
$$

as a function of $x_{4}$ for several values of $R / \rho$, for the background of Eq.(4). ( $\mathcal{N}_{C S}$ is the Chern-Simons number.) See Fig.1. It is clearly seen that at $R / \rho \geq 10$ the topological structure is well separated and localised at the two instanton centers, while for small $R / \rho \leq 1$ the gauge field evidently collapses to some insignificant fluctuation around zero. In the latter situation it is expected that the level crossing [24], hence the fermion number violation, cannot occur.

An analogous observation for the Abelian Higgs model was made in [25]. 
Spectrum of the Dirac operators in the valley background

It is quite remarkable that the dominance of the fermion number violating term (Eq.(14)) at large instanton -antiinstanton separation, occurs without there being a single, dominant mode of the Dirac operators $\bar{D}$ or $D$. The result Eq.(13) for $\operatorname{det} \bar{D}$ neither implies the existence of a particular eigenmode with eigenvalue, $\sim \rho^{2} / R^{3}$, nor requires that such a mode should dominate over others.

Indeed, for any finite $R$ we can establish the following. First of all, no exact zero mode satisfying $\bar{D} \psi=0$ or $D \bar{\psi}=0$ exists. (This can be shown by a direct calculation [19].) Secondly, there are many non-zero modes, definitely lying below $\rho^{2} / R^{3}$. They form a continuous spectrum, reaching down to 0 , if we work in the whole of $\mathbf{R}^{4}$. In particular, for the lowest lying modes with $k \ll \rho^{2} / R^{3}$, both the eigenvalue and the wave function differ little from the free spectrum.

This last point can be made more explicit by putting the system in a large box of size $L^{4}$ such that

$$
L \gg R, \rho
$$

and by applying the standard perturbation theory, treating the valley background of Eq.(4) as a perturbation. We may conveniently consider the Hermitian "Hamiltonian" $\bar{D} D$ or $D \bar{D}$. For the lowest-lying levels and to first order, we find:

$$
\begin{gathered}
E^{(0)}=\mathbf{n}^{2}(\pi / L)^{2}, \quad\left(\mathbf{n}=\left(n_{1}, n_{2}, n_{3}, n_{4}\right) ; n_{j}=1,2, . .\right) ; \\
\left|E^{(1)}\right| \leq \text { const. } \frac{\rho}{L^{3}} \ll E^{(0)} .
\end{gathered}
$$

It can be readily verified that the wave functions are also modified only by a small amount.

(In passing, this shows that a single $i-a$ pair in itself cannot lead to chiral symmetry breaking in QCD: the latter necessarily requires [26] an accumulation of eigenvalues of $D, \bar{D}$ towards 0 . In the context of instanton physics, that would require something like the "instanton liquid" (Ref.[27]).)

The results such as Eq.(13) and Eq.(14) are thus collective effects in which many modes contribute together. No single mode plays any particular role.

\section{An apparent paradox and its resolution.}

We then seem to face a paradox. At large instanton antiinstanton separation, physics must factorise in certain sense, and we do find results (e.g., Eq.(14)) consistent with such intuition. This is fine. The problem is that the mathematics to achieve this looks very different from that of the usual instanton physics where a single fermion zero mode plays a special role. In our case, there is no hint even of the presence of a quasi zero mode (Eq.(17)). What is going on?

The key to the resolution of this apparent paradox is the inequality, (16). In order to be able to compute the S-matrix elements from the four point function (LSZ procedure), we are indeed forced to work in a spacetime region whose linear size $(L)$ is much greater than both of the physical parameters $R$ and $\rho$, independently 
of the ratio $R / \rho$. All fields must be normalised in such a box with an appropriate boundary condition. It is clear that this system (with the $i-a$ background) cannot be connected smoothly to the system with a single (say) antiinstanton inside the box. The gauge field topology remains firmly in the trivial sector.

In spite of our use of the "zero modes" $\eta_{0}^{(a)}, \bar{\zeta}_{0}^{(i)}$ as a convenient device for calculation, they are not good approximate wave functions for the lowest modes in the valley background, however large $R / \rho$ might be. In fact, had one insisted upon using $\eta_{0}^{(a)}$ as the "unperturbed" wave function, one would have discovered that the effect of $\bar{C}$ was always nonperturbative and large (near $x_{i}$ ). *

All this is to be distinguished clearly from the situation where we ask e.g., what the effects of an instanton on a distant planet are. Such a case would correspond to the inequality opposite to (16)

$$
R \gg L
$$

if we restrict ourselves to the instanton-antiinstanton case. As long as we are interested in physics inside our laboratory (or on the earth, anyway) both physics and mathematics are described by just ignoring the distant instanton, to a good approximation. (Particles are produced and detected inside a box of volume, $L^{4} \ll R^{4}$, the gauge field having the winding number -1 , fields and functional integrations defined in the same box, etc.) The effect of the distant instanton is a true, and negligibly small, perturbation in this case.

Fermion number violation in high energy scattering processes.

As is well-known, taking into account the contribution of the Higgs field in the action and using the saddle point approximation in the integrations over the instanton collective coordinates, one finds a relation among the c.m. energy $\sqrt{s}$ and the saddle point values of the parameters $R$ and $\rho$. In a simple toy-model calculation (the second of of Ref.[12]) which uses the valley trajectory of Eq.(4), it was found that at an energy of the order of the sphaleron mass the total action (at the saddle point) vanishes and that the 't Hooft suppression factor disappears.

But at that point, the corresponding saddle point values of the instanton parameters are found to be $\rho=0 ; R / \rho=0$ (hence $A_{\mu}=0$ ) : the unsuppressed cross section should simply correspond to a non-anomalous, perturbative cross section. This was pointed out by some authors [13] also.

What happens is that the anomalous term of Eq.(14) rapidly disappears at $R / \rho \sim 1$ as discussed above, precisely where the valley action sharply drops to

* The standard perturbation theory applied to $H=D \bar{D}=D^{(a)} \bar{D}^{(a)}+H^{\prime}$ with $H^{\prime}=D^{(a)} \bar{C}+C \bar{D}^{(a)}+C \bar{C}$, yields $\Delta E=0$ to all orders, reflecting the topological stability of the fermion zero mode. (Another way to see this is to notice a supersymmetric structure underlying the system. We thank C.Imbimbo for pointing out this to us.) Such a result, however, is false in the case of topologychanging modification of the gauge field. 
zero [15]. We must conclude that the toy-model considered there shows no sign of anomalous cross section (associated with the production of large number $(\sim 1 / \alpha)$ of gauge and Higgs bosons) becoming large, contrary to the original claim.

Of course, there is no proof that all sorts of quantum corrections to the instanton-induced process (1) are correctly described by a classical background such as Eq.(4). But then there are as yet no calculations anyway which show that the anomalous process becomes observable in high energy scatterings.

More generally, the results of this paper imply that, in order for the fermionnumber violating cross sections to become observable at high energies, a new mechanism must be found in which the background field governing the elastic amplitude

Eq.(2) does not effectively reduce to $A_{\mu}^{\text {eff }}=0$. Note that this is necessary, whether or not multiple-instanton type configurations become important at some energies. It is difficult to envisage such a novel mechanism, not accompanied by some finite fraction of the 't Hooft factor.

\section{Theories with no fermions.}

It is often stated that fermions are not essential, as dynamical effects induced by instantons can well be studied in a theory without fermions. This is certainly true, but it does not mean that the consideration of this paper is irrelevant in such a case.

Quite the contrary. The crucial factor,

$$
\exp \left(i k_{j} \cdot x_{a}\right) \exp \left(-i k_{j} \cdot x_{i}\right) \quad(j=1,2)
$$

associated with the external particles, appears upon LSZ amputation applied to the product of the zero modes of Eq.(14) in the case of processes with fermions. In the case of external gauge bosons, the same factor emerges as a result of the "semiclassical" approximation,

$$
\begin{aligned}
& <T A_{\mu}(x) A_{\nu}(u) A_{\rho}(y) A_{\sigma}(v)> \\
& \simeq<T A_{\mu}^{(a)}(x) A_{\nu}^{(a)}(u) A_{\rho}^{(i)}(y) A_{\sigma}^{(i)}(v)>+\ldots
\end{aligned}
$$

It is however clear that this approximation, which is good at large $R / \rho$, fails at $R / \rho<1$ just as Eq.(14) does. The first term of Eq.(20), the anomalous term, disappears precisely when the cross section is claimed to become observable.

\section{Acknowledgments}

Discussions with D. Amati, C. Imbimbo, M. Maggiore, H. Leutwyler and V. Zakharov, have been especially helpful. We are also grateful to many other collegues, M.Bertero, U. Bruzzo, M. Mintchev and M. Porrati among them, for useful information. Carlo Musso has collaborated at an early stage of the work. Part of this work was done during a visit to the Theory Division of CERN. One of us (K.K.) thanks the Physics Department of University of Pisa for hospitality. 


\section{References}

[1] N. Manton, Phys.Rev. D28 (1983) 2019.

[2] H. Aoyama and H. Goldberg, Phys. Lett. B188 (1987) 506.

[3] A. Ringwald, Nucl.Phys. B330 (1990) 1; O. Espinosa, Nucl.Phys. B343 (1990) 310.

[4] J. Cornwall, Phys.Lett. B243 (1990) 271.

[5] L. McLerran, A. Vainshtein and M. Voloshin, Phys. Rev. D42 (1990) 180; P. Arnold and L. McLerran, Phys. Rev. D37 (1988) 1020.

[6] M. Porrati, Nucl.Phys. B347 (1990) 371.

[7] S. Khlebnikov, V. Rubakov and P. Tinyakov, Nucl.Phys. B350 (1991) 441.

[8] V. Zakharov, Nucl. Phys. B371 (1992) 637; Nucl. Phys. B353 (1991) 683; Munich preprint, MPI-PAE/PTh 11/91(1991).

[9] G. Veneziano, unpublished note (1990); CERN TH-6399/92 (1992).

[10] P. Arnold and M. Mattis, Phys.Rev. D42 (1990) 1738; Phys.Rev.Lett. 66 (1991) 13; Phys. Rev. D44 (1991) 3650.

[11] A. Mueller, Nucl.Phys. B353 (1991) 44; Columbia preprint CU-TP-548 (1992).

[12] V.V. Khoze and A. Ringwald, Nucl.Phys. B355 (1991) 351; Phys.Lett. 259 (1991) 106; CERN-TH-6082/91.

[13] M. Maggiore and M. Shifman, Nucl. Phys. B365 (1991)161; Nucl. Phys. B371 (1992) 177; TPI-MINN-92/2-T (1992), to appear in Phys. Rev. D.

[14] S.Yu. Khlebnikov, V.A. Rubakov and P.G. Tinyakov, Nucl. Phys. B350 (1991) 441.

[15] V.V. Khoze, J. Kripfganz and A. Ringwald, Phys. Lett. B275 (1992)381; B277 (1992) 496; B279 (1992) 429E.

[16] O.R. Espinosa, Univ. Washington preprint, UW/PT-91-12 (1991).

[17] D. Dyakonov and V. Petrov, Phys. Lett. B275 (1992) 459.

[18] K. Konishi and C. Musso, Univ. of Genoa preprint, GEF-TH-14 ( 1991).

[19] R. Guida and K. Konishi, in preparation.

[20] M. Voloshin, Nucl. Phys. B363 (1991) 425.

[21] I. Balitsky and A. Yung, Phys. Lett. B168 (1986) 113.

[22] A. Yung, Nucl. Phys. B297 (1988) 47.

[23] L. Brown, R. Creamer, D. Carlitz and C. Lee, Phys. Rev. D17 (1978) 1583;

D. Amati, G.C. Rossi and G. Veneziano, Nucl. Phys. B249 (1985) 1.

[24] C.G. Callan, R. Dashen and D.J. Gross, Phys. Rev. D17 (1978) 2717.

[25] M.Shifman, A seminar at Yale (private communication by M.Maggiore).

[26] H. Leutwyler, Bern preprint, BUTP-91-43 (1991).

[27] E.V. Shuryak, Nucl. Phys. B341 (1990) 1; D.I. Dyakonov and V.Yu. Petrov, Nucl. Phys. B272 (1986) 457.

\section{Figure Caption}

Fig.1 $C\left(x_{4}\right)$ of Eq.(15) versus $x_{4} / \rho$ for $R / \rho=10$ (outmost curve), $R / \rho=5, R / \rho=2$ (middle), $R / \rho=1$, and $R / \rho=0.5$ (innermost curve). The instanton and antiinstanton are situated at $(\mathbf{0}, R / 2)$ and at $(\mathbf{0},-R / 2)$, respectively. 